\title{
Tumor mica status predicts the efficacy of immunotherapy with cytokine-induced killer cells for patients with gastric cancer
}

\author{
Yu Chen, Zhi-feng Zhou, Wei-feng Zhu, Gang Chen, Yi Shi, Wan-song Lin, Zeng-qing Guo, Yun-bin Ye* \\ From 30th Annual Meeting and Associated Programs of the Society for Immunotherapy of Cancer (SITC 2015) \\ National Harbor, MD, USA. 4-8 November 2015
}

\section{Background}

Accumulating evidence has demonstrated that cytokineinduced killer (CIK) cell immunotherapy may improve outcomes when used as an adjuvant to current standard treatment. Previous studies showed that cell signaling through MHC I-related Chain A (MICA)-Natural killer group 2, member D (NKG2D) results in CIK cells activation leading to cytolytic activities against tumor cells. In this study, we determine the relationship between the expression of MICA in gastric cancer tumors after D2 gastrectomy and the clinical outcome of a CIK containing adjuvant therapy.

\section{Methods}

From January 2009 to March 2012, ninety-five consecutive patients with gastric cancer after D2 gastrectomy who received adjuvant chemotherapy combined with CIK cell therapy were enrolled (Table 1). The MICA expression of their tumors was determined by immunohistochemistry (IHC). The IHC score of was obtained by adding the intensity and percentage scores.

\section{Results}

The MICA protein was detected mainly at the cell membrane and in the cytoplasm (Fig.1). High-expression of MICA protein, with IHC scores of 5-7, was documented in 38 of 95 tumor samples (40.0\%). The MICA status was significantly association with the age and stage, $\mathrm{p}=0.008$ and $\mathrm{p}=0.023$, respectively (Table 2 ). Phenotypic analysis of NKG2D on in vitro expanded CIK cells showed that the percentage of NKG2D+ in CD3+/CD56 + , CD3-/CD56+, and CD3+/CD8+ cells populations were $97.2 \pm 1.4 \%, 97.9 \pm 1.8 \%$, and $95.6 \pm 2.1 \%$, respectively.
For the 95 patients, the median DFS was 42.0 months, 95\% CI $=40.82-43.18$ months, and median OS was 45.0 months, $95 \% \mathrm{CI}=41.82-48.18$ months, the 3 -year and 4-year DFS rates were $70.5 \%$ and $34.7 \%$, respectively, and the 3-year and 4-year OS rates were $82.1 \%$ and $49.5 \%$, respectively (Fig. 2A). For patient with high MICA expressing tumors the median DFS and OS were longer than for the patients with tumors with low expression of MICA; 46.0 months vs. 41.0 months $(\mathrm{p}=0.027)$, and 48.0 months vs. 42.0 months $(\mathrm{p}=0.031)$, respectively(Fig. 2B, Table 3). In a multivariate analysis, stage and MICA expression were independent prognostic factors for DFS and OS (Table 4).

Table 1

\begin{tabular}{lllllll}
\hline Variable & & $\mathbf{N}$ & $\mathbf{m}$ DFS & p-value & m OS & p-value \\
\hline Sex & Male & 66 & 42.0 & 0.373 & 44.0 & 0.229 \\
& Female & 29 & 42.0 & & 50.0 & \\
\hline Age & $<65$ & 67 & 41.0 & 0.588 & 48.0 & 0.464 \\
& $\geq 65$ & 28 & 43.0 & & 43.0 & \\
\hline Histological & G1-G2 & 48 & 43.0 & 0.480 & 48.0 & 0.556 \\
grade & G3-G4 & 47 & 31.0 & & 43.0 & \\
& II & 44 & 50.0 & $\mathbf{0 . 0 0 1}$ & 51.0 & $\mathbf{0 . 0 0 6}$ \\
\hline Stage & III & 51 & 36.0 & & 41.0 & \\
& Xelox, & 57 & 41.0 & 0.250 & 43.0 & 0.257 \\
Adjuvant & Folfox4 & & & & & \\
Chemotherapy & PF & 38 & 42.0 & & 46.0 & \\
\hline CIK cycles & $<5$ & 54 & 40.0 & $\mathbf{0 . 0 4 6}$ & 42.0 & 0.075 \\
& $\geq 5$ & 41 & 48.0 & & 50.0 & \\
\hline MICA status & High & 38 & 46.0 & $\mathbf{0 . 0 2 7}$ & 48.0 & $\mathbf{0 . 0 3 1}$ \\
& Low & 57 & 41.0 & & 42.0 & \\
\hline
\end{tabular}

Fujian Provincial Cancer Hospital, Fuzhou, China 


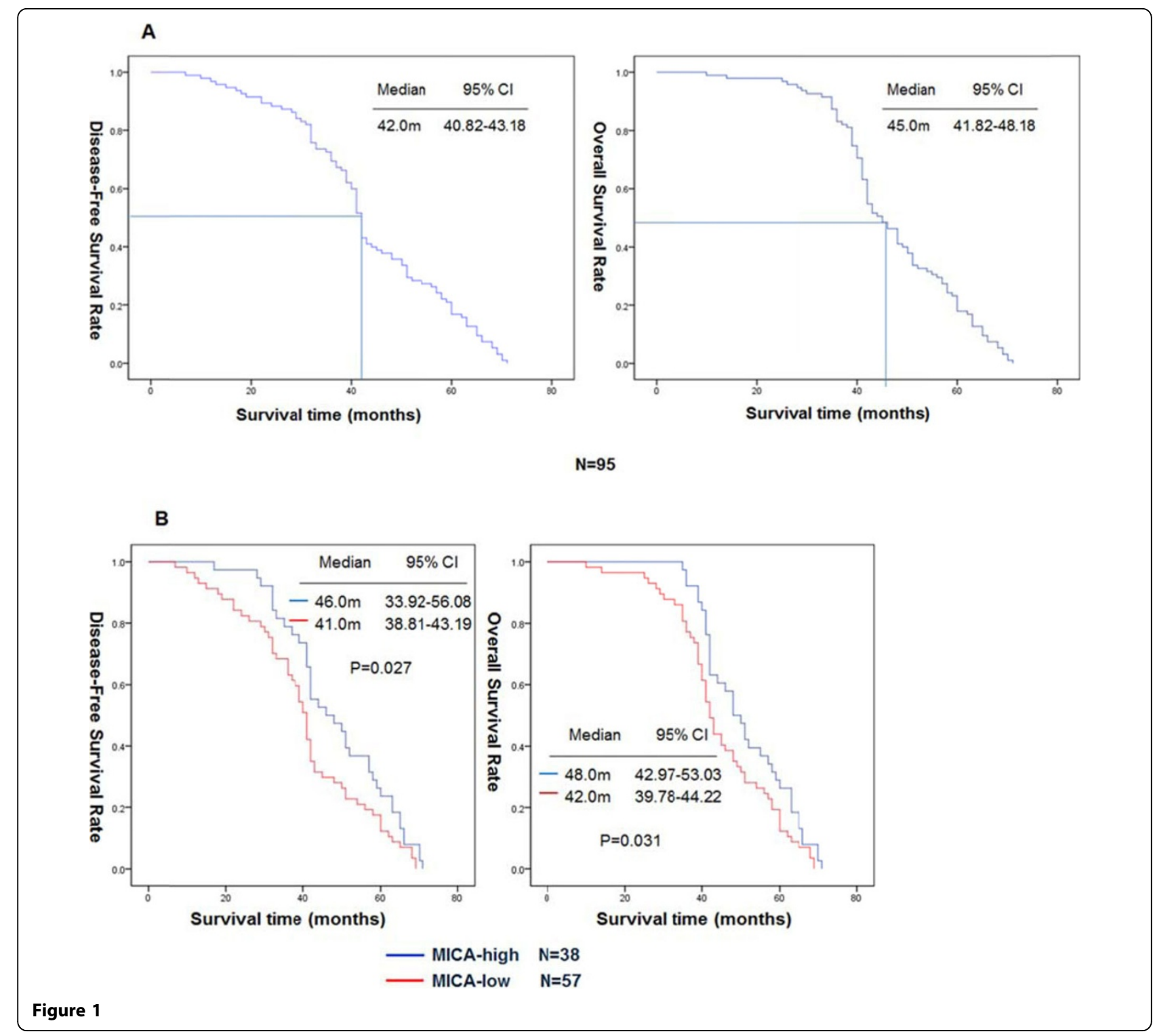

Table 2

\begin{tabular}{|c|c|c|c|c|c|}
\hline Characteristics & & Total95 & MICA highN=38 & MICA lowN=57 & p-value \\
\hline \multirow[t]{2}{*}{ Sex } & Male & 66 & 25 & 41 & 0.524 \\
\hline & Female & 29 & 13 & 16 & \\
\hline \multirow[t]{2}{*}{ Age } & $<65$ & 67 & 21 & 46 & 0.008 \\
\hline & $\geq 65$ & 28 & 17 & 11 & \\
\hline \multirow[t]{2}{*}{ Histological grade } & G1-G2 & 48 & 23 & 25 & 0.111 \\
\hline & G3-G4 & 47 & 15 & 32 & \\
\hline \multirow[t]{2}{*}{ Stage } & $\|$ & 44 & 23 & 21 & 0.023 \\
\hline & III & 51 & 15 & 36 & \\
\hline \multirow[t]{2}{*}{ Adjuvant Chemotherapy } & Xelox, Folfox4 & 57 & 25 & 32 & 0.347 \\
\hline & $\mathrm{PF}$ & 38 & 13 & 25 & \\
\hline \multirow[t]{2}{*}{ CIK cycles } & $<5$ & 54 & 18 & 36 & 0.128 \\
\hline & $\geq 5$ & 41 & 20 & 21 & \\
\hline
\end{tabular}




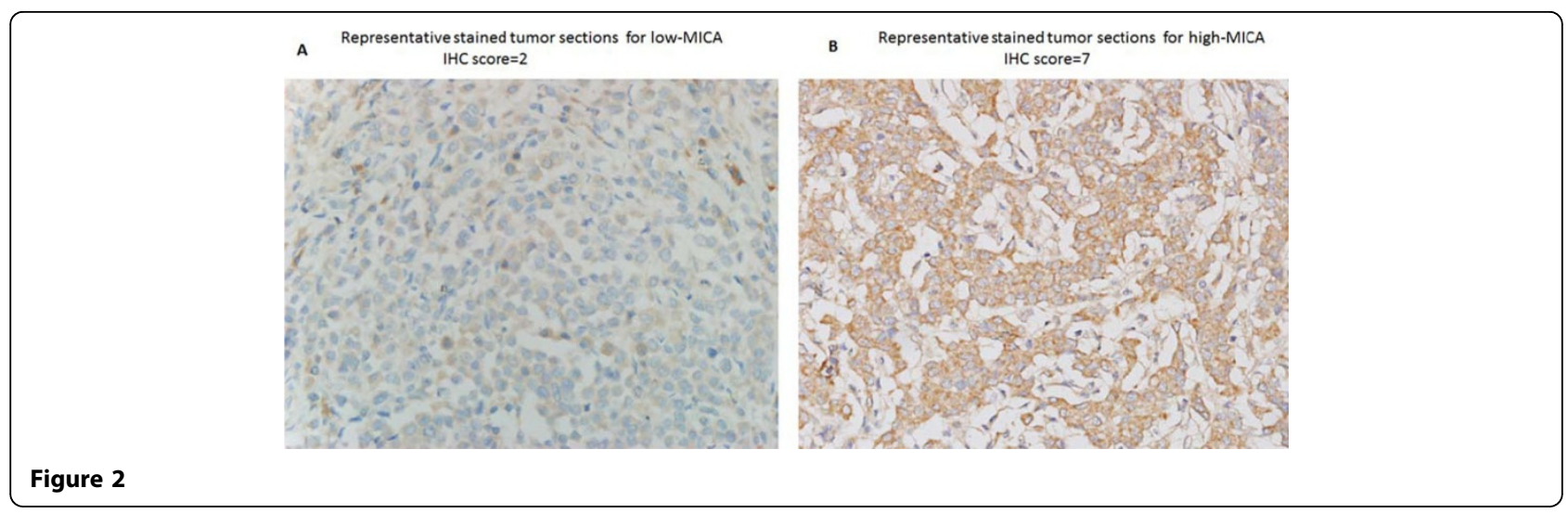

Table 3

\begin{tabular}{|c|c|c|c|}
\hline \multicolumn{2}{|l|}{ Characteristics } & \multirow{2}{*}{$\begin{array}{l}\text { Number } \\
66\end{array}$} & \multirow{2}{*}{$\begin{array}{l}\text { Constituent ratio } \\
69.5 \%\end{array}$} \\
\hline Sex & Male & & \\
\hline & Female & 29 & $30.5 \%$ \\
\hline \multirow[t]{2}{*}{ Age } & $<65$ & 67 & $70.5 \%$ \\
\hline & $\geq 65$ & 28 & $29.5 \%$ \\
\hline \multirow[t]{2}{*}{ Histological grade } & G1-G2 & 48 & $50.5 \%$ \\
\hline & G3-G4 & 47 & $49.5 \%$ \\
\hline \multirow[t]{2}{*}{ Stage } & $\|$ & 44 & $46.3 \%$ \\
\hline & III & 51 & $53.7 \%$ \\
\hline \multirow[t]{2}{*}{ Adjuvant Chemotherapy } & Xelox, Folfox4 & 57 & $60.0 \%$ \\
\hline & $\mathrm{PF}$ & 38 & $40.0 \%$ \\
\hline \multirow[t]{2}{*}{ CIK cycles } & $<5$ & 54 & $56.9 \%$ \\
\hline & $\geq 5$ & 41 & $43.2 \%$ \\
\hline
\end{tabular}

Table 4

\begin{tabular}{|c|c|c|c|c|c|c|}
\hline \multirow[t]{2}{*}{ Variable } & \multicolumn{3}{|l|}{ DFS } & \multicolumn{3}{|l|}{ os } \\
\hline & $P$ value & $\begin{array}{l}\text { Hazard } \\
\text { ratio }\end{array}$ & $95 \% \mathrm{Ci}$ & $P$ value & $\begin{array}{l}\text { Hazard } \\
\text { ratio }\end{array}$ & $95 \% \mathrm{Ci}$ \\
\hline Stage & 0.001 & 1.915 & $\begin{array}{l}1.270- \\
2.886\end{array}$ & 0.010 & 1.713 & $\begin{array}{l}1.138- \\
2.577\end{array}$ \\
\hline MICA & 0.035 & 1.578 & $\begin{array}{l}1.033- \\
2.409\end{array}$ & 0.040 & 1.557 & $\begin{array}{l}1.020- \\
2.376\end{array}$ \\
\hline
\end{tabular}

\section{Conclusion}

Our findings show that adjuvant chemotherapy plus CIK therapy treatment is a promising modality for treating gastric cancer patients after D2 gastrectomy. Especially, those who have tumors with high-expression of MICA were more likely to benefit from such a treatment strategy. Subsequent studies in clinical trial cohorts will be required to confirm the clinical utility of these markers.

Published: 4 November 2015
doi:10.1186/2051-1426-3-S2-P61

Cite this article as: Chen et al:: Tumor mica status predicts the efficacy of immunotherapy with cytokine-induced killer cells for patients with gastric cancer. Journal for ImmunoTherapy of Cancer 2015 3(Suppl 2):P61.

\section{Submit your next manuscript to BioMed Central and take full advantage of:}

- Convenient online submission

- Thorough peer review

- No space constraints or color figure charges

- Immediate publication on acceptance

- Inclusion in PubMed, CAS, Scopus and Google Scholar

- Research which is freely available for redistribution

Submit your manuscript at www.biomedcentral.com/submit 